\title{
Precision Gas System Handbook
}
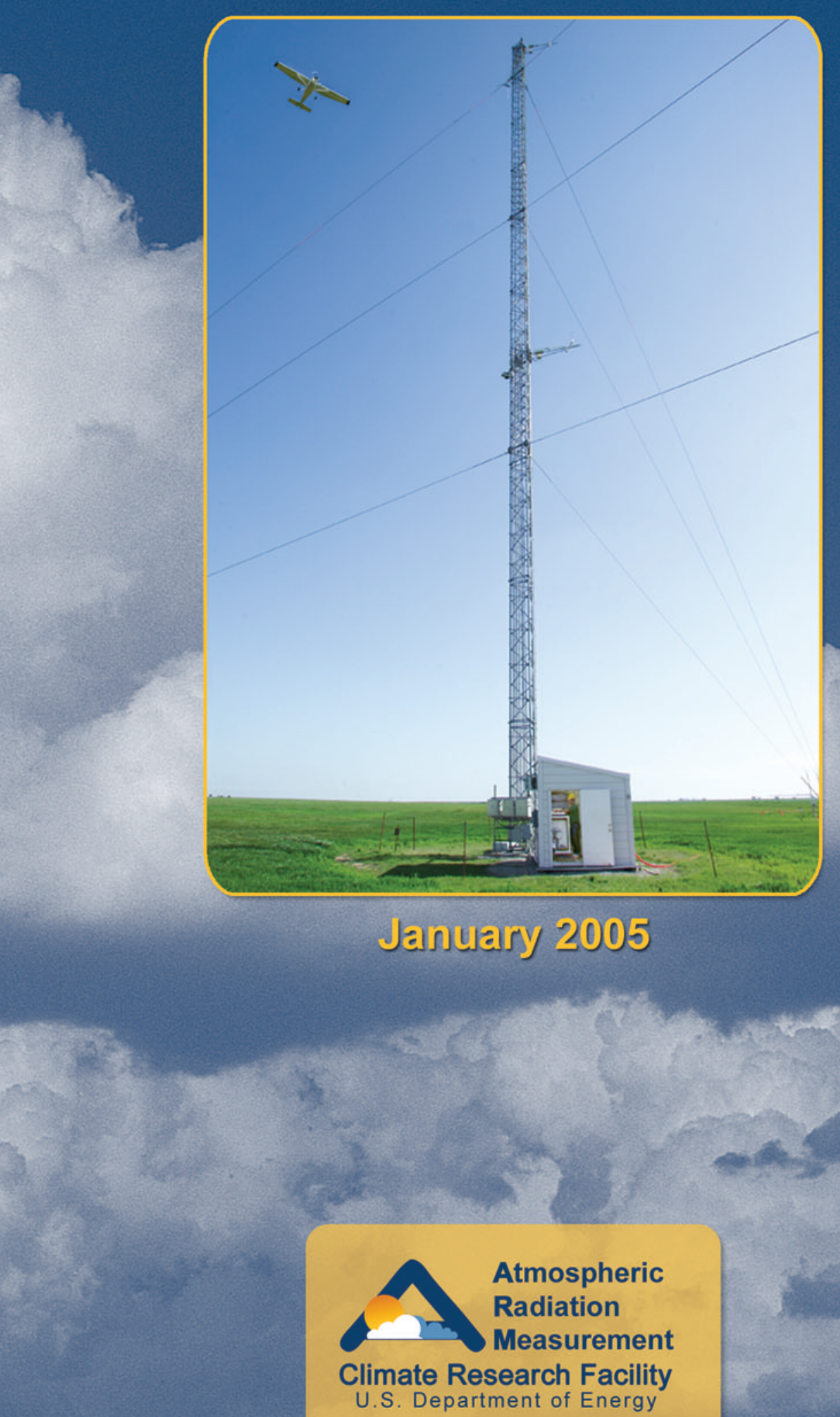

Work supported by the U.S. Department of Energy Office of Science, Office of Biological and Environmental Research 


\section{Precision Gas System (PGS) Handbook}

January 2005

M. Torn

Work supported by the U.S. Department of Energy, Office of Science, Office of Biological and Environmental Research 


\section{Contents}

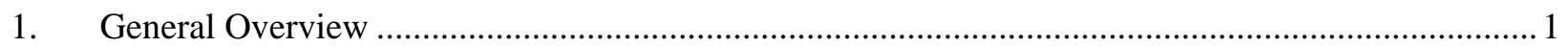

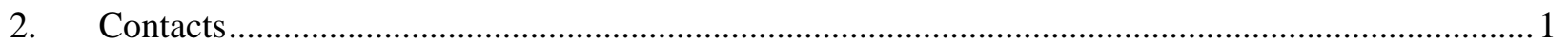

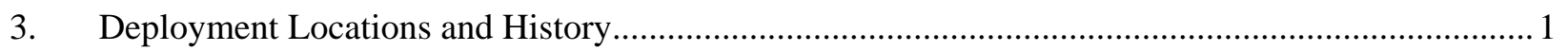

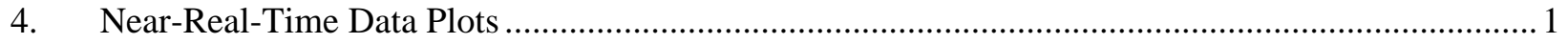

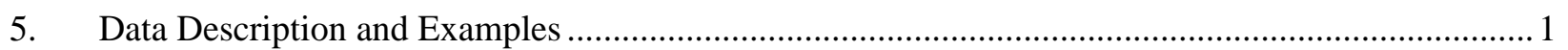

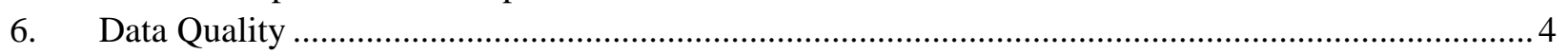

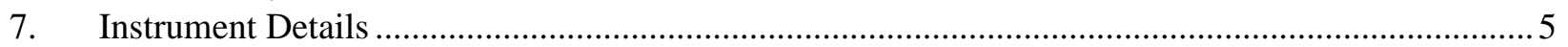




\section{General Overview}

This precision gas system (PGS) makes high-accuracy, high-precision measurements of $\mathrm{CO}_{2}$ mixing ratio (ppmv dry air) in air sampled at 2, 4, 25, and $60 \mathrm{~m}$ above the ground.

\section{Contacts}

\subsection{Mentor}

Margaret Torn, Scientist

Earth Sciences Division

Mail Stop 90-1116

E.O. Lawrence Berkeley National Laboratory

1 Cyclotron Rd.

Berkeley, CA 94720

Phone: 510-495-2223

Fax: 510-486-7070

Email: mstorn@lbl.gov

Website: http://www-esd.lbl.gov/ESD_staff/torn/index.html

\subsection{Instrument Developer}

Lawrence Berkeley National Laboratory (LBNL) staff built the system.

$\mathrm{Li}-\mathrm{Cor}$ is the vendor for the infrared gas analyzer (IRGA), model LI-6252 $\mathrm{CO}_{2}$ Analyzer (see http://www.licor.com/env/Products/GasAnalyzers/li6262/6262.jsp for product description).

\section{Deployment Locations and History}

The precision gas system is located at the base of the $60 \mathrm{~m}$ tower at the Central Facility at the Atmospheric Radiation Measurement (ARM) Program's Climate Research Facility Southern Great Plains (SGP) locale. The system operates continuously, except for occasional power outages.

\section{Near-Real-Time Data Plots}

Data stream 1: Quick views of the precision gas system data for yesterday are available at http://co2anal.lbl.gov/worldview/pgs-yest.html.

Data stream 2: Quick views of the precision gas system data for the past week are available at http://co2anal.lbl.gov/worldview/pgs-week.html.

\section{Data Description and Examples}

\subsection{Data File Contents}

Data are reported as ppm (V) $\mathrm{CO}_{2}$ in dry air. 


\subsubsection{Primary Variables and Expected Uncertainty}

$\mathrm{CO}_{2}$ mixing ratio in dry air.

\subsubsection{Definition of Uncertainty}

Samples are bracketed by certified standards.

\subsubsection{Secondary/Underlying Variables}

This section is not applicable to this instrument.

\subsubsection{Diagnostic Variables}

The diagnostic variables are listed in the table for Data Quality Flags, Section 5.1.4.

\subsubsection{Data Quality Flags}

\begin{tabular}{ll} 
QC flag name & Function (abbreviations defined below) \\
\hline qc_co2mv_ch5 & qc flag on co2mv_ch5 (reference channel) \\
qc_co2mv_slope & qc flag on co2mv_slope \\
$\begin{array}{ll}\text { qc_co2mv_slope_se qc flag on co2mv_slope_se } \\
\text { qc_co2ppm }\end{array}$ & qc flag on co2ppm \\
& \\
qc_co2ppm_25m & qc flag on co2ppm_2m \\
qc_co2ppm_2m & qc flag on co2ppm_2m \\
qc_co2ppm_4m & qc flag on co2ppm_2m \\
qc_co2ppm_60m & qc flag on co2ppm_2m \\
qc_co2stor_25m & qc flag on co2stor_25m \\
qc_co2stor_4m & qc flag on co2stor_4m \\
qc_co2stor_60m & qc flag on co2stor_60m \\
qc_coeff1_se & qc flag on coeff1_se \\
qc_coeff2_se & qc flag on coeff2_se \\
qc_coeff3_se & qc flag on coeff3_se \\
qc_del_co2stor_25m qc flag on del_co2stor_25m \\
qc_del_co2stor_4m & qc flag on del_co2stor_4m \\
qc_del_co2stor_60m qc flag on del_co2stor_60m \\
qc_flow_rate & qc flag on flow rate \\
qc_pres & qc flag on pres \\
qc_pres_ctrlr & qc flag on pres_controller \\
qc_pres1 & qc flag on pres1 \\
qc_pres2 & qc flag on pres2 \\
qc_pres3 & qc flag on pres3
\end{tabular}




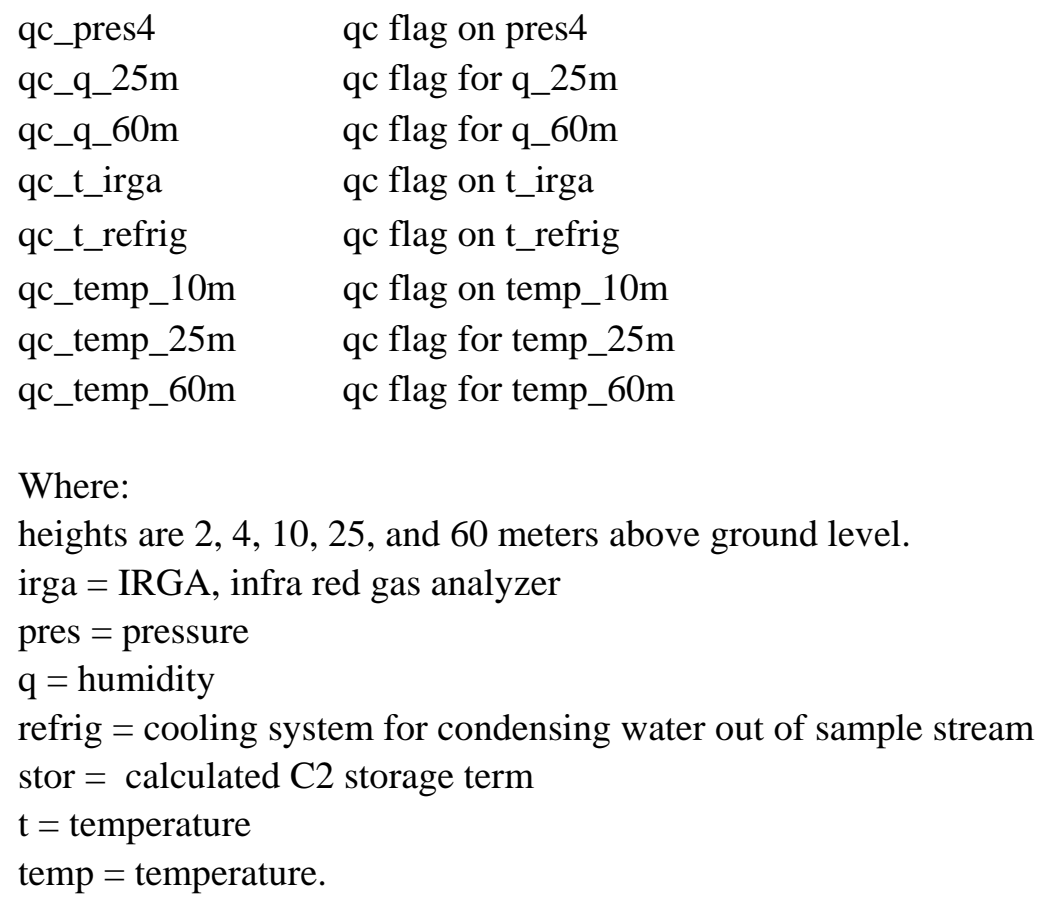

Where:

heights are 2, 4, 10, 25, and 60 meters above ground level.

irga $=$ IRGA, infra red gas analyzer

pres $=$ pressure

$\mathrm{q}=$ humidity

refrig = cooling system for condensing water out of sample stream

stor $=$ calculated $\mathrm{C} 2$ storage term

$\mathrm{t}=$ temperature

temp $=$ temperature .

\title{
5.1.5 Dimension Variables
}

This section is not applicable to this instrument.

\subsection{Annotated Examples}

\author{
QC flag name Function (abbreviations defined below) \\ qc_co2mv_ch5 qc flag on co2mv_ch5 (reference channel) \\ qc_co2mv_slope qc flag on co2mv_slope \\ qc_co2mv_slope_se qc flag on co2mv_slope_se \\ qc_co2ppm qc flag on co2ppm \\ qc_co2ppm_25m qc flag on co2ppm_2m \\ qc_co2ppm_2m qc flag on co2ppm_2m \\ qc_co2ppm_4m qc flag on co2ppm_2m \\ qc_co2ppm_60m qc flag on co2ppm_2m \\ qc_co2stor_25m qc flag on co2stor_25m \\ qc_co2stor_4m qc flag on co2stor_4m \\ qc_co2stor_60m qc flag on co2stor_60m \\ qc_coeff1_se qc flag on coeff1_se \\ qc_coeff2_se qc flag on coeff2_se \\ qc_coeff3_se qc flag on coeff3_se \\ qc_del_co2stor_25m qc flag on del_co2stor_25m \\ qc_del_co2stor_4m qc flag on del_co2stor_4m
}




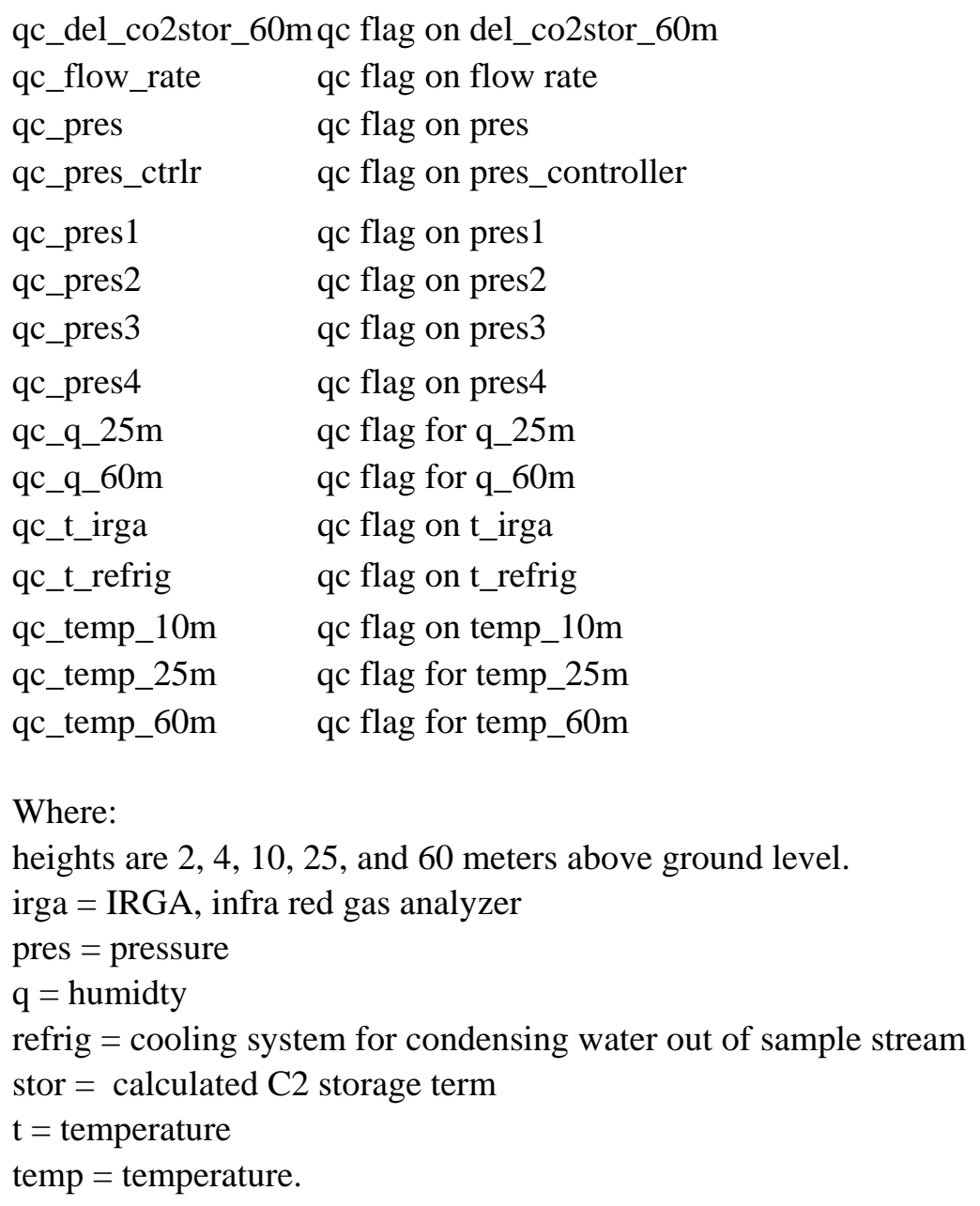

\subsection{User Notes and Known Problems}

Datastreams are available through the ARM Data Archive.

\subsection{Frequently Asked Questions}

Where do I get more information?

Contact the instrument mentor (see Section 2.1).

\section{Data Quality}

\subsection{Data Quality Health and Status}

The instrument has operated continuously since July, 2001. Occasional loss of data has occurred due to loss of site power or other causes (ice storm, pump failure). Please check data quality reports for specifics. 


\subsection{Data Reviews by Instrument Mentor}

ARM staff perform preventive maintenance checks weekly and post-preventive maintenance reports on the internet. ARM carbon scientists at LBNL check these reports each week. We evaluate the zero drift (change in reference gas value) and specifications of all instrument components every week.

Examples of variables that are diagnostic of instrument performance include:

- Temperature of IRGA

- Pressure of gas in IRGA

- Pressure of each air sample supplied to valve manifold

- Temperature of the cooling (drying) unit

- Flow rate of sample gas through the IRGA.

\subsection{Data Assessments by Site Scientist/Data Quality Office}

Not available at this time.

\subsection{Value-Added Procedures and Quality Measurement Experiments}

None at present.

\section{Instrument Details}

\subsection{Detailed Description}

\subsubsection{List of Components}

\begin{tabular}{ll} 
QC flag name & Function (abbreviations defined below) \\
\hline qc_co2mv_ch5 & qc flag on co2mv_ch5 (reference channel) \\
qc_co2mv_slope & qc flag on co2mv_slope \\
$\begin{array}{ll}\text { qc_co2mv_slope_se qc flag on co2mv_slope_se } \\
\text { qc_co2ppm }\end{array}$ & qc flag on co2ppm \\
qc_co2ppm_25m & qc flag on co2ppm_2m \\
qc_co2ppm_2m & qc flag on co2ppm_2m \\
qc_co2ppm_4m & qc flag on co2ppm_2m \\
qc_co2ppm_60m & qc flag on co2ppm_2m \\
qc_co2stor_25m & qc flag on co2stor_25m \\
qc_co2stor_4m & qc flag on co2stor_4m \\
qc_co2stor_60m & qc flag on co2stor_60m \\
qc_coeff1_se & qc flag on coeff1_se \\
qc_coeff2_se & qc flag on coeff2_se \\
qc_coeff3_se & qc flag on coeff3_se
\end{tabular}




\begin{tabular}{|c|c|}
\hline \multicolumn{2}{|c|}{$\begin{array}{l}\text { qc_del_co2stor_25m qc flag on del_co2stor_25m } \\
\text { qc_del_co2stor_4m qc flag on del_co2stor_4m } \\
\text { qc_del_co2stor_60m qc flag on del_co2stor_60m }\end{array}$} \\
\hline qc_flow_rate & qc flag on flow rate \\
\hline qc_pres & qc flag on pres \\
\hline qc_pres_ctrlr & qc flag on pres_controller \\
\hline qc_pres1 & qc flag on pres1 \\
\hline qc_pres2 & qc flag on pres2 \\
\hline qc_pres3 & qc flag on pres3 \\
\hline qc_pres4 & qc flag on pres4 \\
\hline qc_q_25m & qc flag for q_25m \\
\hline qc_q_60m & qc flag for q_60m \\
\hline qc_t_irga & qc flag on t_irga \\
\hline qc_t_refrig & qc flag on t_refrig \\
\hline qc_temp_10m & qc flag on temp_10m \\
\hline qc_temp_25m & qc flag for temp_25m \\
\hline qc_temp_60m & qc flag for temp_60m \\
\hline
\end{tabular}

Where:

heights are 2, 4, 10, 25, and 60 meters above ground level.

irga $=$ IRGA, infra red gas analyzer

pres $=$ pressure

$\mathrm{q}=$ humidty

refrig $=$ cooling system for condensing water out of sample stream

stor $=$ calculated C2 storage term

$\mathrm{t}=$ temperature

temp $=$ temperature.

Infra-Red Gas Analyzer (IRGA), LI-6252 (see http://env.licor.com/):

- Sample is dried with a cooling unit and a Nafion membrane drier.

- Flow is regulated with mass flow controller, pumps, and valves.

- A 1-liter buffer volume is used to dampen very-short-term variation in $\mathrm{CO}_{2}$ concentration.

- System is directed by a datalogger (Campbell 23x) that downloads to a computer every day.

Data collection system uses a 2.8 GHz Intel PC clone; operating system Windows 2000.

\subsubsection{System Configuration and Measurement Methods}

We measure one sample for 3 minutes at a time; the reported value is the average of the last 30 seconds.

The IRGA is run in differential mode, with a reference gas (ca $360 \mathrm{ppm}$ ) through the reference cell at all times. Every 15 minutes, we analyze the reference gas through the sample cell as well, to correct for gain (zero drift) in the system. Every 4 hours, we run four calibration standards (National Oceanic and Atmospheric Administration Climate Monitoring Diagnostics Laboratory (NOAA CMDL) certified 
standards at approximately 330, 360, 390, and $420 \mathrm{ppm}$ ). Sample $\mathrm{CO}_{2}$ concentration is calculated with the standards run before and after it.

We have followed protocols developed by the NOAA CMDL (e.g., Bakwin et al.) for their high precision, continuous $\mathrm{CO}_{2}$ monitoring instruments.

\subsubsection{Specifications}

This section is not applicable to this instrument.

\subsection{Theory of Operation}

See Section 7.1, Detailed Description.

\subsection{Calibration}

\subsubsection{Theory and Procedures}

See above.

\subsubsection{History}

Before deployment at ARM SGP, we did a blind comparison of a tank of $\mathrm{CO}_{2}$ in air with the NOAACMDL standards lab. Our measurement agreed with theirs to $0.05 \mathrm{ppm}$.

Currently the instrument performance is checked against weekly NOAA CMDL flask samples collected from the 60-m air-sampling inlet.

\subsection{Operation and Maintenance}

\subsubsection{User Manual}

This section is not applicable to this instrument.

\subsubsection{Routine and Corrective Maintenance Documentation}

ARM staff perform preventive maintenance checks weekly and post-preventive maintenance reports on the internet. ARM carbon scientists at LBNL check these reports each week. We evaluate the zero drift (change in reference gas value) and specifications of all instrument components every week. For examples of instrument operations that we manage through the Routine and Corrective Maintenance documents, See Data Reviews by Instrument Mentor.

\subsubsection{Software Documentation}

There is none at this time. 


\subsubsection{Additional Documentation}

There is none at this time.

\subsection{Glossary}

See the ARM Glossary.

\subsection{Acronyms}

ARM: Atmospheric Radiation Measurement (Program)

CMDL: Climate Monitoring Diagnostics Laboratory

IRGA: infrared gas analyzer

LBNL: Lawrence Berkeley National Laboratory

NOAA: National Oceanic and Atmospheric Administration

PGS: precision gas system

SGP: Southern Great Plains

Also see the ARM Acronyms and Abbreviations.

\subsection{Citable References}

Bakwin P.S., P.P. Tans, C.L. Zhao, W. Ussler, and E. Quesnell. 1995. Measurements of carbon dioxide on a very tall tower. Tellus Series B-Chemical and Physical Meteorology, V47(N5):535-549.

Bakwin, P.S., P.P. Tans, D.F. Hurst, and C.L. Zhao. 1998. Measurements of carbon dioxide on very tall towers: results of the NOAA/CMDL program. Tellus Series B-Chemical and Physical Meteorology, V50(N5):401-415.

Tans P.P., P.S. Bakwin, and D.W. Guenther. 1996. A Feasible global carbon cycle observing system - a plan to decipher todays carbon cycle based on observations. Global Change Biology, V2(N3):309-318. 